\title{
PROXIMAL FEMORAL EPIPHYSIOLYSIS AND SUBCLINICAL HYPOTHYROIDISM: CASE REPORT
}

\section{ABSTRACT}

Proximal femoral epiphysiolysis is an orthopedic disease that is prevalent during adolescence, because this coincides with the time of greatest growth of osteomuscular structures. Curiously, some patients present this disease early, and this outcome converts to the possible etiological explanation that the slippage might occur through a growth spurt. For these patients, the genesis of the slippage has not yet been elucidated, but endocrine disorders have been noted as possible causes. In an attempt to strengthen the theory of endocrinological etiology and present the results from surgical treatment for this pathological condition, the case of a male patient aged 9 years and 3 months with proximal femoral epiphysiolysis and subclinical hypothyroidism who was diagnosed and treated at our university's teaching hospital is reported here.

Keywords - Epiphyses, Slipped; Hypothyroidism; Bone Screws; Child

The aim of this study was to add to the literature an unusual case of epiphysiolysis involving a child with subclinical hypothyroidism, since few studies have dealt with the intersection of these pathological conditions.

\section{CASE REPORT}

The patient was a male child aged nine years and three months who came to the pediatric orthopedic clinic with a complaint of pain in both knees, which worsened after doing physical activities at school. In his previous medical history, there was no record of delayed development or underlying pathological conditions. Physical examination showed that he was overweight (body mass index, BMI, of 26.3). No secondary sexual characteristics were encountered.

The patient scored 82 points on the Harris scale for hip diseases. Orthopedic physical examination in dorsal decubitus showed positioning of external rotation of the left hip. A mobility test showed elevated external rotation and limitation of internal rotation of the left lower limb. No abnormality was observed on the right side.

1 - Undergraduate Medical Student ( $11^{\text {th }}$ semester $)$ at the Lutheran University of Brazil, Canoas, RS, Brazil.

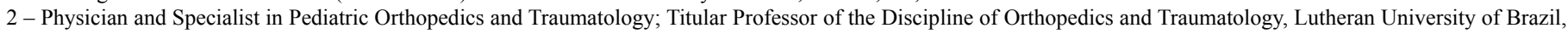
Canoas, RS, Brazil.

Work performed at the University Hospital of the Lutheran University of Brazil, Canoas, RS.

Correspondence: Av. Bento Gonçalves 1515, ap. 1809/A, Bairro Parthenon, 90650-002 Canoas, RS, Brazil. E-mail: grazyymello@hotmail.com

Work received for publication: September 13, 2011; accepted for publication: October 25, 2011.

The authors declare that there was no conflict of interest in conducting this work 
The Trendelenburg sign was absent. There was no evidence of atrophy of the quadriceps or reduction of the length of the affected limb. The neurological physical examination was normal. Because of the orthopedic examination suggestive of slippage of the femoral head, we requested x-rays in AP + Lowenstein. The patient returned with the radiographic images, which were suggestive of proximal epiphysiolysis of the femoral head, in varus, on his left side (Figure 1).

Endocrinological screening was then performed in an attempt to explain why this slippage had occurred at such an early age, given that this was an overweight patient with a family history of hypothyroidism. The results from the laboratory tests showed triglycerides of 236 (normal: 150), TSH of 7.58 (normal range: 0.27 to 4.2 ) and free $\mathrm{T} 4$ within the normal range.

The surgical correction indicated consisted of fixation with a since cannulated screw on the left side, including contralateral prophylactic pinning (Figure 2). After the operation, the patient reported grade 3 on the visual analogue pain scale. At a follow-up one after the operation, the patient was already performing physical activities in a normal manner, without complaints of functional limitation or pain, and his Harris score was 95 . He was referred to the endocrinology service, where his follow-up is continuing, presenting normal $\mathrm{TH}$ levels with hormone replacement.

\section{DISCUSSION}

Even thought the etiology of PEF is uncertain, it seems to include hormonal involvement in its genesis. Because of this, there is a tendency to proceed

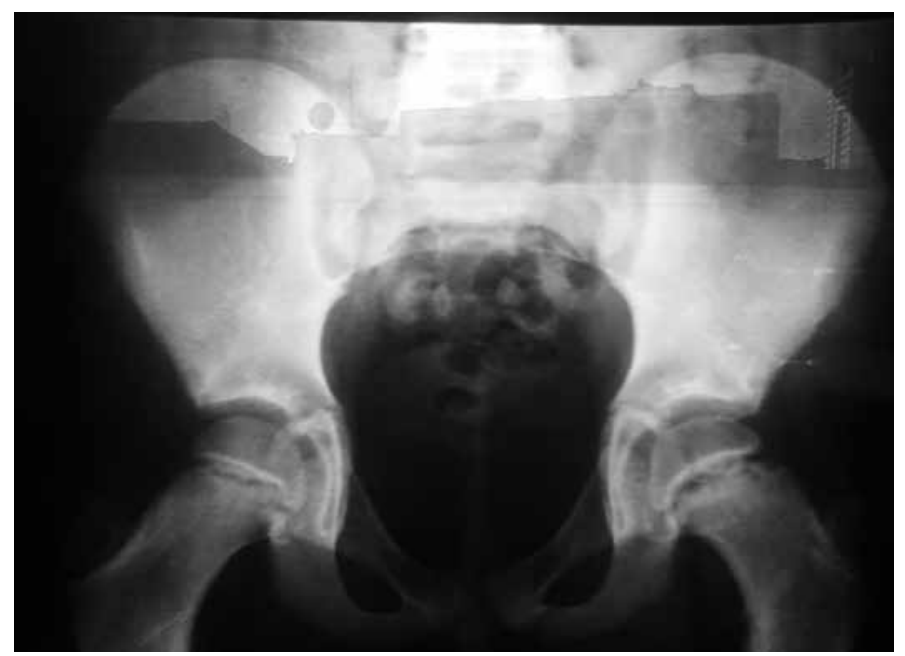

Figure 1 - Preoperative radiograph showing proximal epiphysiolysis on the left side.

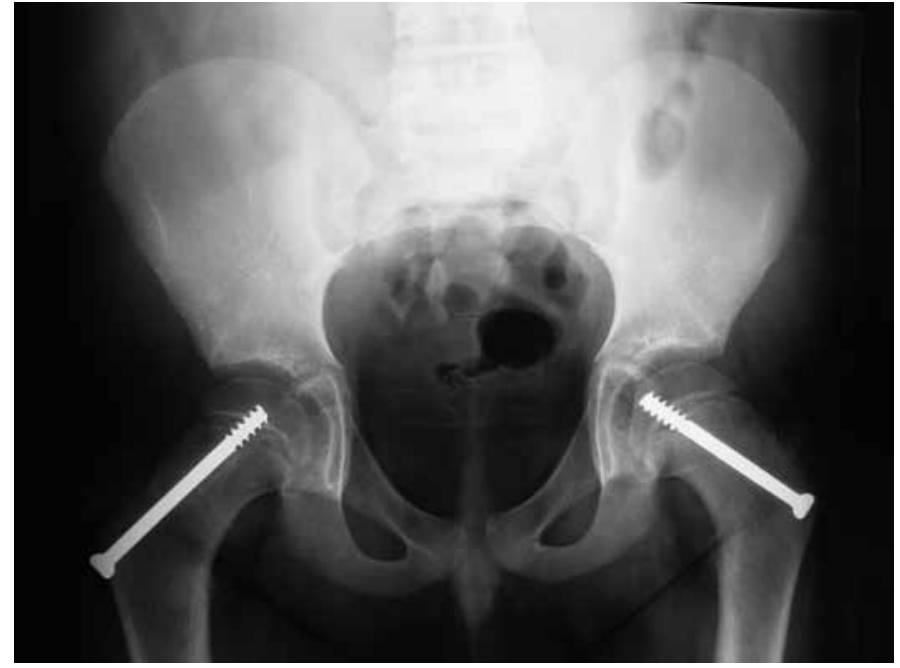

Figure 2 - In situ bilateral fixation, seen on postoperative radiograph.

with endocrinological investigation when the diagnosis is confirmed during childhood, given that this is an unusual occurrence before puberty. To form the diagnostic hypothesis, tests to evaluate the thyroid and lipid profile were requested, because the patient was overweight. Curiously, elevated TSH was observed alone, which suggested that the hypothyroidism was subclinical, given that the serum T4 levels were within the normal range. There is little discussion of such findings in the literature, but the few such authors emphasize that alterations in TSH levels, even if occurring in isolation, may be responsible for growth plate anomalies $^{(5)}$, since these may influence the skeletal remodeling through interaction with the specific receptors expressed in bone cells ${ }^{(6)}$. We did not find any studies conclusively associating PEF with subclinical hypothyroidism in children, although there are some studies that make a connection with classical hypothyroidism.

Regarding symptoms, pain in the hip and/or knee region, with or without accompanying claudication of the affected limb, is the commonest complaint among patients with PEF. This condition should always be considered in the differential diagnosis when a child or adolescent presents this type of discomfort ${ }^{(7)}$. Concordant with the literature, we found that pain was the reason why the patient sought medical care. This finding, together with the propaedeutic and radiological findings, helped us in making the diagnostic hypothesis. Nonetheless, for investigating the definitive diagnosis of PEF, it is essential not only to perform a rigorous physical examination on the hip, but also to using imaging examinations. To make the radiographic diagnosis 
of epiphysiolysis, anteroposterior views of the pelvis in neutral and Lowenstein positions were used ${ }^{(8)}$.

For surgical correction of PEF, different techniques have been described. The indication is based on the degree and duration of slippage, the degree of cartilage lesion and the experience of the center involved. Thus, there is no definitive surgical protocol. Nevertheless, it is accepted that the objectives in treating PEF are basically to stimulate closure of the growth plate, achieve stabilization, prevent new slippage and maintain the anatomofunctional organization of the bone structures involved, so that there is no impairment of growth ${ }^{(9)}$. In this context, in situ fixation using a screw is the treatment most used for less severe cases of slippage, and good results have been observed with this technique, even in children under the age of ten years ${ }^{(3)}$. Based on the literature and on experience at our clinic, fixation with a single cannulated screw without osteotomy is the preferred treatment. After one year of follow-up, we observed that there was good correction of the slippage, with improvement in the physical examination. There was no evidence of deleterious effects such as avascular necrosis or osteochondrolysis. Removal of the screw was not indicated, since our patient is still within the growth phase, and it could cause recurrence of the slippage.

Based on the high incidence of contralateral occurrence, especially in patients with endocrinological disorders, and the higher frequency of bilaterality among patients under the age of ten years ${ }^{(3)}$, it was decided to perform prophylactic fixation of the contralateral hip. No abnormalities in the physical examination or radiological examination were observed one year after the surgery.

In this report, we used the Harris score ${ }^{(10)}$, since this is considered to be a good predictor of the impact of hip limitations. The patient obtained a score of 82 at the preoperative consultation, which denotes mild impairment of day-to-day activities. After the surgical intervention, the score obtained was 95 , which is considered to be excellent on this scale. This allows us to infer that the surgical treatment was effective from the functional point of view, with consequent improvement in the patient's quality of life.

It could be seen that there was greater concern in the literature regarding the forms of treatment for $\mathrm{PEF}$, given that relatively few results relating to its genesis and physiopathological mechanisms have been published, especially regarding occurrences in children. Nonetheless, in the light of the characteristics of the patient presented here and patients in the literature, it can be understood that value needs to be placed on the endocrinological etiology, especially through searching for subclinical alterations. Because this is a case report, it can only be suggested that the screening for pediatric patients diagnosed with PEF should also include laboratory tests on the thyroid. Studies with larger samples are needed in order to confirm that this hypothesis may form part of the etiological characteristics of this pathological condition.

\section{REFERÊNCIAS}

1. Tayton K. Does the upper femoral epiphysis slip or rotate? J Bone Joint Surg Br. 2007;89(10):1402-6.

2. Tachdjian MO. Clinical pediatric orthopedics: the art of diagnosis and principles of management. Stamford, Connecticut: Appleton and Lange; 1997.

3. Atri LJ, González AOC, Aziz JJ, Castañeda LP.Slipped femoral epiphysis in children under 10 years of age. Clinical and radiologic evaluation of the surgical treatment. Acta Ortop Mex. 2009;23(4):213-6

4. Biondi B, Cooper DS. The clinical significance of subclinical thyroid dysfunction. Endocr Rev. 2008;29(1):76-131.

5. Sun L, Davies TF, Blair HC, Abe E, Zaidi M. TSH and bone loss. Ann N Y Acad Sci. 2006;1068:309-18.

6. Abe E, Marians RC, Yu W, Wu XB, Ando T, Li Y, et al. TSH is a negative

regulator of skeletal remodeling. Cell. 2003;115(2):151-62.

7. Solduk L, Sogut O, Kaya H, Gokdemir MT, Ozkanli U. An adolescent patient with hip pain: slipped capital femoral epiphysis. J Clin Med Res. 2011;3(2):99-100

8. Weigall P, Vladusic S, Torode I. Slipped upper femoral epiphysis in children-delays to diagnosis. Aust Fam Physician. 2010;39(3):151-3

9. Falciglia F, Aulisa AG, Giordano M, Boldrini R, Guzzanti V. Slipped capital femoral epiphysis: an ultrastructural study before and after osteosynthesis. Acta Orthop. 2010;81(3):331-6

10. Harris WH. Traumatic arthritis of the hip after dislocation and acetabular fractures: treatment by mold arthroplasty. An end-result study using a new method of result evaluation. J Bone Joint Surg Am. 1969;51(4):737-55. 